\title{
Correction to: An in vitro mechanistic approach towards understanding the distinct pathways regulating insulin resistance and adipogenesis by apocynin
}

\author{
Sai Bharadwaja ${ }^{l}$, Praveen Kumar Issac ${ }^{l}$, Jocelyn Cleta ${ }^{l}$, \\ Rakesh Jeganaathan $^{1}$, Sri Snehaa Chandrakumar ${ }^{2}$ and \\ SuJATHA Sundaresan ${ }^{1} *$ (ii \\ ${ }^{1}$ Department of Biotechnology, School of Bioengineering, SRM Institute of Science and Technology, \\ Kattankulathur, Kancheepuram District, Tamil Nadu 603 203, India \\ ${ }^{2}$ Department of Biotechnology, Anna University, BIT Campus, Tiruchirappalli, Tamil Nadu 620024 , \\ India \\ *Corresponding author (Email, sujathas1@srmist.edu.in)
}

Correction to: J Biosci (2021) 46:008 https://doi.org/10.1007/s12038-020-00134-2

In the 2021 issue of the Journal of Biosciences in the article titled "An in vitro mechanistic approach towards understanding the distinct pathways regulating insulin resistance and adipogenesis by apocynin” by Sai Bharadwaja, Praveen Kumar Issac, Jocelyn Cleta, Rakesh Jeganathan, Sri Snehaa Chandrakumar and Sujatha Sundaresan (https://doi.org/10.1007/s12038-020-00134-2; Vol. 46, Article No. 008), the author Rakesh Jeganaathan's name was incorrectly mentioned as "Rakesh Jeganathan". The correct name should read as "Rakesh Jeganaathan”.

The original article can be found online at https://doi.org/10.1007/s12038-020-00134-2. 